УДК 519.652

\title{
Construction of Interpolation Splines Minimizing the Semi-norm in the Space $K_{2}\left(P_{m}\right)$
}

\author{
Abdullo R. Hayotov* \\ V.I. Romanovskiy Institute of Mathematics \\ Uzbekistan Academy of Sciences \\ M. Ulugbek street, 81, Tashkent, 100125
}

Uzbekistan

Received 07.10.2017, received in revised form 10.12.2017, accepted 22.03.2018

\begin{abstract}
In the present paper, using S.L. Sobolev's method, interpolation splines that minimize the expression $\int_{0}^{1}\left(\varphi^{(m)}(x)+\omega^{2} \varphi^{(m-2)}(x)\right)^{2} d x$ in the space $K_{2}\left(P_{m}\right)$ are constructed. Explicit formulas for the coefficients of the interpolation splines are obtained. The obtained interpolation splines are exact for monomials $1, x, x^{2}, \ldots, x^{m-3}$ and for trigonometric functions $\sin \omega x$ and $\cos \omega x$.

Keywords: interpolation spline, Hilbert space, norm minimizing property, Sobolev's method, discrete argument function.

DOI: $10.17516 / 1997-1397-2018-11-3-383-396$.
\end{abstract}

\section{Introduction. Statement of the Problem}

In order to find an approximate representation of a function $\varphi$ by elements of a certain finite dimensional space, it is possible to use values of this function at some finite set of points $x_{\beta}$, $\beta=0,1, \ldots, N$. The corresponding problem is called the interpolation problem, and the points $x_{\beta}$ are called the interpolation nodes.

There are polynomial and spline interpolations. It is known that the polynomial approximation is non-practical for approximation of functions with finite and little smoothness, which often occurs in applications. This circumstance makes it necessary to work with splines. Spline functions are very useful in applications. Classes of spline functions possess many nice structural properties as well as excellent approximation powers. They are used, for example, in data fitting, function approximation, numerical quadrature, and the numerical solution of ordinary and partial differential equations, integral equations, and so on. Many books are devoted to the theory of splines, for example, Ahlberg et al [1], Arcangeli et al [2], Attea [3], Berlinet and ThomasAgnan [4], Bojanov et al [5], de Boor [7], Eubank [10], Green and Silverman [13], Ignatov and Pevniy [21], Korneichuk et al [23], Laurent [24], Mastroianni and Milovanović [26], Nürnberger [27], Schumaker [29], Stechkin and Subbotin [36], Vasilenko [37], Wahba [38] and others.

If the exact values $\varphi\left(x_{\beta}\right)$ of an unknown smooth function $\varphi(x)$ at the set of points $\left\{x_{\beta}, \beta=\right.$ $0,1, \ldots, N\}$ in an interval $[a, b]$ are known, it is usual to approximate $\varphi$ by minimizing

$$
\int_{a}^{b}\left(g^{(m)}(x)\right)^{2} d x
$$

*hayotov@mail.ru

(c) Siberian Federal University. All rights reserved 
in the set of interpolating functions (i.e., $\left.g\left(x_{\beta}\right)=\varphi\left(x_{\beta}\right), \beta=0,1, \ldots, N\right)$ of the space $L_{2}^{(m)}(a, b)$. Here $L_{2}^{(m)}(a, b)$ is the Sobolev space of functions with a square integrable $m$-th generalized derivative. It turns out that the solution is a natural polynomial spline of degree $2 m-1$ with knots $x_{0}, x_{1}, \ldots, x_{N}$ called the interpolating $D^{m}$-spline for the points $\left(x_{\beta}, \varphi\left(x_{\beta}\right)\right)$. In the non periodic case this problem has been first investigated by Holladay [20] for $m=2$. His results have been generalized by de Boor [6] for any $m$. In the Sobolev space $\widetilde{L_{2}^{(m)}}$ of periodic functions, the minimization problem of integrals of the type (1.1) was investigated in works [11, 12, 14, 25, 28] and others.

We consider the Hilbert space

$$
K_{2}\left(P_{m}\right)=\left\{\varphi:[0,1] \rightarrow \mathbb{R} \mid \varphi^{(m-1)} \text { is absolutely continuous and } \varphi^{(m)} \in L_{2}(0,1)\right\},
$$

equipped with the norm

$$
\left\|\varphi \mid K_{2}\left(P_{m}\right)\right\|=\left\{\int_{0}^{1}\left(P_{m}\left(\frac{\mathrm{d}}{\mathrm{d} x}\right) \varphi(x)\right)^{2} \mathrm{~d} x\right\}^{1 / 2},
$$

where

$$
P_{m}\left(\frac{\mathrm{d}}{\mathrm{d} x}\right)=\frac{\mathrm{d}^{m}}{\mathrm{~d} x^{m}}+\omega^{2} \frac{\mathrm{d}^{m-2}}{\mathrm{~d} x^{m-2}}, \quad \omega>0, \quad m \geqslant 2
$$

and

$$
\int_{0}^{1}\left(P_{m}\left(\frac{\mathrm{d}}{\mathrm{d} x}\right) \varphi(x)\right)^{2} \mathrm{~d} x<\infty
$$

The equality (1.2) is the semi-norm, and $\|\varphi\|=0$ if and only if $\varphi(x)=c_{1} \sin \omega x+c_{2} \cos \omega x+$ $R_{m-3}(x)$, where $R_{m-3}(x)$ is a polynomial of degree $m-3$.

It should be noted that for a linear differential operator of order $n, L \equiv P_{n}(\mathrm{~d} / \mathrm{d} x)$, Ahlberg, Nilson, and Walsh in the book [1, Chapter 6] investigated the Hilbert spaces in the context of generalized splines. Namely, with the inner product

$$
\langle\varphi, \psi\rangle=\int_{0}^{1} L \varphi(x) \cdot L \psi(x) \mathrm{d} x,
$$

$K_{2}\left(P_{n}\right)$ is a Hilbert space if we identify functions that differ by a solution of $L \varphi=0$.

Consider the following interpolation problem:

Problem 1. To find the function $S_{m}(x) \in K_{2}\left(P_{m}\right)$, which gives the minimum of the norm (1.2) and satisfies the interpolation condition

$$
S_{m}\left(x_{\beta}\right)=\varphi\left(x_{\beta}\right), \quad \beta=0,1, \ldots, N,
$$

where $x_{\beta} \in[0,1]$ are the nodes of interpolation, $\varphi\left(x_{\beta}\right)$ are given values.

Following [37, p.46, Theorem 2.2] we get the analytic representation of the interpolation spline $S_{m}(x)$

$$
S_{m}(x)=\sum_{\gamma=0}^{N} C_{\gamma} G_{m}\left(x-x_{\gamma}\right)+d_{1} \sin (\omega x)+d_{2} \cos (\omega x)+R_{m-3}(x),
$$

where $C_{\gamma}, \gamma=0,1, \ldots, N, d_{1}$ and $d_{2}$ are real numbers, $R_{m-3}(x)=\sum_{\alpha=0}^{m-3} r_{\alpha} x^{\alpha}$ is a polynomial of degree $m-3$ and

$$
G_{m}(x)=\frac{(-1)^{m} \operatorname{sign} x}{4 \omega^{2 m-1}}\left((2 m-3) \sin \omega x-\omega x \cos \omega x+2 \sum_{k=1}^{m-2} \frac{(-1)^{k}(m-k-1)(\omega x)^{2 k-1}}{(2 k-1) !}\right)
$$


is a fundamental solution of the operator $\frac{\mathrm{d}^{2 m}}{\mathrm{~d} x^{2 m}}+2 \omega^{2} \frac{\mathrm{d}^{2 m-2}}{\mathrm{~d} x^{2 m-2}}+\omega^{4} \frac{\mathrm{d}^{2 m-4}}{\mathrm{~d} x^{2 m-4}}$, i.e., $G_{m}(x)$ is a solution of the equation

$$
G_{m}^{(2 m)}(x)+2 \omega^{2} G_{m}^{(2 m-2)}(x)+\omega^{4} G_{m}^{(2 m-4)}(x)=\delta(x)
$$

here $\delta(x)$ is Dirac's delta function.

It is known that (see, for instance, [37]) the solution $S_{m}(x)$ of the form (1.4) of Problem 1 exists, is unique when $N+1 \geqslant m$ and coefficients $C_{\gamma}, d_{1}, d_{2}$ and $r_{\alpha}$ of $S_{m}(x)$ are defined by the following system of $N+m+1$ linear equations

$$
\begin{gathered}
\sum_{\gamma=0}^{N} C_{\gamma} G_{m}\left(x_{\beta}-x_{\gamma}\right)+d_{1} \sin \left(\omega x_{\beta}\right)+ \\
d_{2} \cos \left(\omega x_{\beta}\right)+R_{m-3}\left(x_{\beta}\right)=\varphi\left(x_{\beta}\right), \quad \beta=0,1, \ldots, N, \\
\sum_{\gamma=0}^{N} C_{\gamma} \sin \left(\omega x_{\gamma}\right)=0, \\
\sum_{\gamma=0}^{N} C_{\gamma} \cos \left(\omega x_{\gamma}\right)=0, \\
\sum_{\gamma=0}^{N} C_{\gamma} x_{\gamma}^{\alpha}=0, \quad \alpha=0,1, \ldots, m-3 .
\end{gathered}
$$

The main aim of the present paper is to solve Problem 1, i.e., to solve system (1.7)-(1.10) for equally spaced nodes $x_{\beta}=h \beta, \beta=0,1, \ldots, N, h=1 / N, N+1 \geqslant m$ and to find analytic formulas for the coefficients $C_{\gamma}, d_{1}, d_{2}$ and $r_{\alpha}$ of $S_{m}(x)$.

It should be noted that interpolation splines minimizing the semi-norms in the $L_{2}^{(m)}(0,1)$, $W_{2}^{(m, m-1)}(0,1)$ and $K_{2}\left(P_{2}\right)$ Hilbert spaces were constructed in works $[8,17,18,19,31,32]$ by using Sobolev's method. Furthermore, the connection between interpolation spline and optimal quadrature formula in the sense of Sard in $L_{2}^{(m)}(0,1)$ and $K_{2}\left(P_{2}\right)$ spaces were shown in [8] and [18].

The rest of the paper is organized as follows: in Section 2 we give some definitions and known results. In Section 3 we give the algorithm for solution of system (1.7)-(1.10) when the nodes $x_{\beta}$ are equally spaced. Using this algorithm, the coefficients of the interpolation spline $S_{m}(x)$ are computed in Section 4.

\section{Preliminaries}

In this section we give some definitions and known results that we need to prove the main results.

Below we mainly use the concept of discrete argument functions and operations on them. The theory of discrete argument functions is given in [34, 35]. For completeness we give some definitions about functions of discrete argument.

Assume that the nodes $x_{\beta}$ are equally spaced, i.e., $x_{\beta}=h \beta, h=\frac{1}{N}, N=1,2, \ldots$.

Definition 2.1. The function $\varphi(h \beta)$ is a function of discrete argument if it is given on some set of integer values of $\beta$. 
Definition 2.2. The inner product of two discrete functions $\varphi(h \beta)$ and $\psi(h \beta)$ is given by

$$
[\varphi(h \beta), \psi(h \beta)]=\sum_{\beta=-\infty}^{\infty} \varphi(h \beta) \cdot \psi(h \beta),
$$

if the series on the right hand side of the last equality converges absolutely.

Definition 2.3. The convolution of two functions $\varphi(h \beta)$ and $\psi(h \beta)$ is the inner product

$$
\varphi(h \beta) * \psi(h \beta)=[\varphi(h \gamma), \psi(h \beta-h \gamma)]=\sum_{\gamma=-\infty}^{\infty} \varphi(h \gamma) \cdot \psi(h \beta-h \gamma) .
$$

The Euler-Frobenius polynomials $E_{k}(x), k=1,2, \ldots$ are defined by the following formula $[35]$

$$
E_{k}(x)=\frac{(1-x)^{k+2}}{x}\left(x \frac{d}{d x}\right)^{k} \frac{x}{(1-x)^{2}}
$$

$E_{0}(x)=1$.

For the Euler-Frobenius polynomials $E_{k}(x)$ the following identity holds

$$
E_{k}(x)=x^{k} E_{k}\left(\frac{1}{x}\right)
$$

and also the following theorem is true

Theorem 2.1 (Lemma 3 of [30]). Polynomial $Q_{k}(x)$ which is defined by the formula

$$
Q_{k}(x)=(x-1)^{k+1} \sum_{i=0}^{k+1} \frac{\Delta^{i} 0^{k+1}}{(x-1)^{i}}
$$

is the Euler-Frobenius polynomial (2.1) of degree $k$, i.e. $Q_{k}(x)=E_{k}(x)$, where $\Delta^{i} 0^{k}=$ $\sum_{l=1}^{i}(-1)^{i-l} C_{i}^{l} l^{k}$

The following formula is valid [15]:

$$
\sum_{\gamma=0}^{n-1} q^{\gamma} \gamma^{k}=\frac{1}{1-q} \sum_{i=0}^{k}\left(\frac{q}{1-q}\right)^{i} \Delta^{i} 0^{k}-\left.\frac{q^{n}}{1-q} \sum_{i=0}^{k}\left(\frac{q}{1-q}\right)^{i} \Delta^{i} \gamma^{k}\right|_{\gamma=n}
$$

where $\Delta^{i} \gamma^{k}$ is the finite difference of order $i$ of $\gamma^{k}, q$ is the ratio of a geometric progression. When $|q|<1$ from (2.4) we have

$$
\sum_{\gamma=0}^{\infty} q^{\gamma} \gamma^{k}=\frac{1}{1-q} \sum_{i=0}^{k}\left(\frac{q}{1-q}\right)^{i} \Delta^{i} 0^{k}
$$

In our computations we need the discrete analogue $D_{m}(h \beta)$ of the differential operator $\frac{d^{2 m}}{d x^{2 m}}+$ $2 \omega^{2} \frac{d^{2 m-2}}{d x^{2 m-2}}+\omega^{4} \frac{d^{2 m-4}}{d x^{2 m-4}}$ which satisfies the following equality

$$
D_{m}(h \beta) * G_{m}(h \beta)=\delta(h \beta),
$$


where $G_{m}(h \beta)$ is the discrete argument function corresponding to $G_{m}(x)$ defined by $(1.5), \delta(h \beta)$ is equal to 0 when $\beta \neq 0$ and is equal to 1 when $\beta=0$, i.e. $\delta(h \beta)$ is the discrete delta-function. The equation (2.6) is the discrete analogue of the equation (1.6).

In $[16,17]$ the discrete analogue $D_{m}(h \beta)$ of the differential operator $\frac{d^{2 m}}{d x^{2 m}}+2 \omega^{2} \frac{d^{2 m-2}}{d x^{2 m-2}}+$ $\omega^{4} \frac{d^{2 m-4}}{d x^{2 m-4}}$, which satisfies equation $(2.6)$, is constructed and the following is proved.

Theorem 2.2. The discrete analogue to the differential operator $\frac{d^{2 m}}{d x^{2 m}}+2 \omega^{2} \frac{d^{2 m-2}}{\mathrm{~d} x^{2 m-2}}+\omega^{4} \frac{d^{2 m-4}}{d x^{2 m-4}}$ satisfying equation (2.6) has the form

$$
D_{m}(h \beta)=p \begin{cases}\sum_{k=1}^{m-1} A_{k} \lambda_{k}^{|\beta|-1}, & |\beta| \geqslant 2, \\ 1+\sum_{k=1}^{m-1} A_{k}, & |\beta|=1, \\ C+\sum_{k=1}^{m-1} \frac{A_{k}}{\lambda_{k}}, & \beta=0\end{cases}
$$

where

$$
\begin{gathered}
A_{k}=\frac{\left(1-\lambda_{k}\right)^{2 m-4}\left(\lambda_{k}^{2}-2 \lambda_{k} \cos h \omega+1\right)^{2} p_{2 m-2}^{(2 m-2)}}{\lambda_{k} \mathcal{P}_{2 m-2}^{\prime}\left(\lambda_{k}\right)} \\
C=4-4 \cos h \omega-2 m-\frac{p_{2 m-3}^{(2 m-2)}}{p_{2 m-2}^{(2 m-2)}, \quad p=\frac{2 \omega^{2 m-1}}{(-1)^{m} p_{2 m-2}^{(2 m-2)}},} \\
p_{2 m-2}^{(2 m-2)}=(2 m-3) \sin h \omega-h \omega \cos h \omega+2 \sum_{k=1}^{m-2} \frac{(-1)^{k}(m-k-1)(h \omega)^{2 k-1}}{(2 k-1) !} \\
\mathcal{P}_{2 m-2}(x)=\sum_{s=0}^{2 m-2} p_{s}^{(2 m-2)} x^{s}=(1-x)^{2 m-4}\left[[(2 m-3) \sin h \omega-h \omega \cos h \omega] x^{2}+\right. \\
+[2 h \omega-(2 m-3) \sin (2 h \omega)] x+[(2 m-3) \sin h \omega-h \omega \cos h \omega]]+ \\
+2\left(x^{2}-2 x \cos h \omega+1\right)^{2} \sum_{k=1}^{m-2} \frac{(-1)^{k}(m-k-1)(h \omega)^{2 k-1}(1-x)^{2 m-2 k-4} E_{2 k-2}(x)}{(2 k-1) !}
\end{gathered}
$$

here $E_{2 k-2}(x)$ is the Euler-Frobenius polynomial of degree $2 k-2, \omega>0, h \omega \leqslant 1, h=1 / N$, $N \geqslant m-1, m \geqslant 2, p_{2 m-2}^{(2 m-2)}, p_{2 m-3}^{(2 m-2)}$ are the coefficients and $\lambda_{k}$ are the roots of the polynomial $\mathcal{P}_{2 m-2}(\lambda),\left|\lambda_{k}\right|<1$.

Furthermore several properties of the discrete argument function $D_{m}(h \beta)$ were given in [16, 17]. Here we give the following properties of the discrete argument function $D_{m}(h \beta)$ which we need in our computations.

Theorem 2.3. The discrete analogue $D_{m}(h \beta)$ of the differential operator $\frac{d^{2 m}}{d x^{2 m}}+2 \omega^{2} \frac{d^{2 m-2}}{d x^{2 m-2}}+$ $\omega^{4} \frac{d^{2 m-4}}{d x^{2 m-4}}$ satisfies the following equalities
1) $D_{m}(h \beta) * \sin (h \omega \beta)=0$,
2) $D_{m}(h \beta) * \cos (h \omega \beta)=0$,
3) $D_{m}(h \beta) *(h \omega \beta) \sin (h \omega \beta)=0$,
4) $D_{m}(h \beta) *(h \omega \beta) \cos (h \omega \beta)=0$,
5) $D_{m}(h \beta) *(h \beta)^{\alpha}=0, \alpha=0,1, \ldots, 2 m-5$. 


\section{The algorithm for computation of coefficients of interpolation splines}

In the present section we give the algorithm for solution of system (1.7)-(1.10) when the nodes $x_{\beta}$ are equally spaced, i.e., $x_{\beta}=h \beta, h=\frac{1}{N}, N=1,2, \ldots$. Here we use a method similar to the one suggested by S. L. Sobolev $[33,35]$ for finding the coefficients of optimal quadrature formulas in the Sobolev space $L_{2}^{(m)}(0,1)$.

Suppose that $C_{\beta}=0$ when $\beta<0$ and $\beta>N$. Using Definition 2.3, we rewrite the system (1.7)-(1.10) in the convolution form

$$
\begin{gathered}
G_{m}(h \beta) * C_{\beta}+d_{1} \sin (h \omega \beta)+d_{2} \cos (h \omega \beta)+R_{m-3}(h \beta)=\varphi(h \beta), \quad \beta=0,1, \ldots, N, \\
\sum_{\beta=0}^{N} C_{\beta} \cdot \sin (h \omega \beta)=0, \\
\sum_{\beta=0}^{N} C_{\beta} \cdot \cos (h \omega \beta)=0, \\
\sum_{\beta=0}^{N} C_{\beta} \cdot(h \beta)^{\alpha}=0, \quad \alpha=0,1, \ldots, m-3,
\end{gathered}
$$

where $R_{m-3}(h \beta)=\sum_{\alpha=0}^{m-3} r_{\alpha}(h \beta)^{\alpha}$.

Thus we have the following problem.

Problem 2. Find the coefficients $C_{\beta},(\beta=0,1, \ldots, N), d_{1}, d_{2}$ and polynomial $R_{m-3}(h \beta)$ of degree $m-3$ which satisfy the system (3.1)-(3.4).

Further on we investigate Problem 2 which is equivalent to Problem 1. Instead of $C_{\beta}$ we introduce the following functions

$$
\begin{aligned}
& v(h \beta)=G_{m}(h \beta) * C_{\beta}, \\
& u(h \beta)=v(h \beta)+d_{1} \sin (h \omega \beta)+d_{2} \cos (h \omega \beta)+R_{m-3}(h \beta) .
\end{aligned}
$$

Now we express the coefficients $C_{\beta}$ using the function $u(h \beta)$.

Taking into account (2.7), (3.6) and Theorems 2.2, 2.3, for the coefficients we have

$$
C_{\beta}=D_{m}(h \beta) * u(h \beta) .
$$

Thus, if we find the function $u(h \beta)$, then the coefficients $C_{\beta}$ will be found from equality (3.7)

To calculate the convolution (3.7) it is required to find the representation of the function $u(h \beta)$ for all integer values of $\beta$. From equality (3.1) we get that $u(h \beta)=\varphi(h \beta)$ when $h \beta \in[0,1]$. Now we need to find the representation of the function $u(h \beta)$ when $\beta<0$ and $\beta>N$.

Since $C_{\beta}=0$ when $h \beta \notin[0,1]$, we have

$$
C_{\beta}=D_{m}(h \beta) * u(h \beta)=0, \quad h \beta \notin[0,1] .
$$

Now we calculate the convolution $v(h \beta)=G_{m}(h \beta) * C_{\beta}$ when $\beta \leqslant 0$ and $\beta \geqslant N$. 
Suppose $\beta \leqslant 0$ then taking into account equalities (1.5), (3.2)-(3.4), we have

$$
\begin{aligned}
v(h \beta)= & \sum_{\gamma=-\infty}^{\infty} C_{\gamma} G_{m}(h \beta-h \gamma) \sum_{\gamma=0}^{N} C_{\gamma} \frac{(-1)^{m} \operatorname{sign}(h \beta-h \gamma)}{4 \omega^{2 m-1}}[(2 m-3) \sin (h \omega \beta-h \omega \gamma)- \\
& \left.-(h \omega \beta-h \omega \gamma) \cos (h \omega \beta-h \omega \gamma)+2 \sum_{k=1}^{m-2} \frac{(-1)^{k}(m-k-1)(h \omega \beta-h \omega \gamma)^{2 k-1}}{(2 k-1) !}\right]= \\
= & -\frac{(-1)^{m}}{4 \omega^{2 m-1}}\left[\cos (h \omega \beta) \sum_{\gamma=0}^{N} C_{\gamma}(h \omega \gamma) \cos (h \omega \gamma)+\sin (h \omega \beta) \sum_{\gamma=0}^{N} C_{\gamma}(h \omega \gamma) \sin (h \omega \gamma)+\right. \\
& \left.+2 \sum_{k=\left[\frac{m}{2}\right]}^{m-2} \sum_{\alpha=m-2}^{2 k-1} \frac{(-1)^{k+\alpha}(m-k-1) \omega^{2 k-1}}{(2 k-1-\alpha) ! \alpha !}(h \beta)^{2 k-1-\alpha} \sum_{\gamma=0}^{N} C_{\gamma}(h \gamma)^{\alpha}\right],
\end{aligned}
$$

where $\left[\frac{m}{2}\right]$ is the integer part of $\frac{m}{2}$.

Thus when $\beta \leqslant 0$ we get

$$
v(h \beta)=-D_{1} \sin (h \omega \beta)-D_{2} \cos (h \omega \beta)-Q_{m-3}(h \beta),
$$

where

$$
D_{1}=\frac{(-1)^{m}}{4 \omega^{2 m-1}} \sum_{\gamma=0}^{N} C_{\gamma}(h \omega \gamma) \sin (h \omega \gamma), \quad D_{2}=\frac{(-1)^{m}}{4 \omega^{2 m-1}} \sum_{\gamma=0}^{N} C_{\gamma}(h \omega \gamma) \cos (h \omega \gamma)
$$

and

$$
Q_{m-3}(h \beta)=\frac{(-1)^{m}}{2 \omega^{2 m-1}} \sum_{k=\left[\frac{m}{2}\right]}^{m-2} \sum_{\alpha=m-2}^{2 k-1} \frac{(-1)^{k+\alpha}(m-k-1) \omega^{2 k-1}}{(2 k-1-\alpha) ! \alpha !}(h \beta)^{2 k-1-\alpha} \sum_{\gamma=0}^{N} C_{\gamma}(h \gamma)^{\alpha}
$$

is an unknown polynomial of degree $m-3$ of $(h \beta)$.

Similarly, in the case $\beta \geqslant N$ for the convolution $v(h \beta)=G_{m}(h \beta) * C_{\beta}$ we obtain

$$
v(h \beta)=D_{1} \sin (h \omega \beta)+D_{2} \cos (h \omega \beta)+Q_{m-3}(h \beta),
$$

We denote

$$
\begin{array}{ccc}
R_{m-3}^{-}(h \beta)=R_{m-3}(h \beta)-Q_{m-3}(h \beta), & d_{1}^{-}=d_{1}-D_{1}, & d_{2}^{-}=d_{2}-D_{2} \\
R_{m-3}^{+}(h \beta)=R_{m-3}(h \beta)+Q_{m-3}(h \beta), & d_{1}^{+}=d_{1}+D_{1}, & d_{2}^{+}=d_{2}+D_{2}
\end{array}
$$

where $R_{m-3}^{-}(h \beta)=\sum_{\alpha=0}^{m-3} r_{\alpha}^{-} \cdot(h \beta)^{\alpha}, R_{m-3}^{+}(h \beta)=\sum_{\alpha=0}^{m-3} r_{\alpha}^{+} \cdot(h \beta)^{\alpha}$.

Taking into account (3.6), (3.8) and (3.11) we get the following problem

Problem 3. Find a solution of the equation

$$
D_{m}(h \beta) * u(h \beta)=0, \quad h \beta \notin[0,1]
$$

having the form:

$$
u(h \beta)= \begin{cases}d_{1}^{-} \sin (h \omega \beta)+d_{2}^{-} \cos (h \omega \beta)+R_{m-3}^{-}(h \beta), & \beta \leqslant 0, \\ \varphi(h \beta), & 0 \leqslant \beta \leqslant N, \\ d_{1}^{+} \sin (h \omega \beta)+d_{2}^{+} \cos (h \omega \beta)+R_{m-3}^{+}(h \beta), & \beta \geqslant N .\end{cases}
$$

Here $R_{m-3}^{-}(h \beta)$ and $R_{m-3}^{+}(h \beta)$ are unknown polynomials of degree $m-3$ with respect to $h \beta$. 
If we find $d_{1}^{-}, d_{1}^{+}, d_{2}^{-}, d_{2}^{+}$and polynomials $R_{m-3}^{-}(h \beta), R_{m-3}^{+}(h \beta)$ then from $(3.12),(3.13)$ we have

$$
\begin{aligned}
& R_{m-3}(h \beta)=\frac{1}{2}\left(R_{m-3}^{+}(h \beta)+R_{m-3}^{-}(h \beta)\right), \quad d_{k}=\frac{1}{2}\left(d_{k}^{+}+d_{k}^{-}\right), \quad k=1,2, \\
& Q_{m-3}(h \beta)=\frac{1}{2}\left(R_{m-3}^{+}(h \beta)-R_{m-3}^{-}(h \beta)\right), \quad D_{k}=\frac{1}{2}\left(d_{k}^{+}-d_{k}^{-}\right), \quad k=1,2 .
\end{aligned}
$$

Unknowns $d_{1}^{-}, d_{1}^{+}, d_{2}^{-}, d_{2}^{+}$and polynomials $R_{m-3}^{-}(h \beta), R_{m-3}^{+}(h \beta)$ can be found from equation (3.14), using the function $D_{m}(h \beta)$ defined by (2.7). Then we obtain explicit form of the function $u(h \beta)$ and from (3.7) we find the coefficients $C_{\beta}$. Furthermore from (3.16) we get $R_{m-3}(h \beta), d_{1}$ and $d_{2}$.

Thus, Problem 3 and respectively Problems 2 and 1 will be solved.

In the next section we apply this algorithm to compute the coefficients $C_{\beta}, \beta=0,1, \ldots, N$, $d_{1}, d_{2}$ and $r_{\alpha}, \alpha=0,1, \ldots, m-3$ of the interpolation spline (1.4) for any $m \geqslant 2$ and $N+1 \geqslant m$.

\section{Computation of coefficients of interpolation spline (1.4)}

In this section, using the above algorithm, we obtain the explicit formulas for the coefficients of the interpolation spline (1.4) which, as we have proved in the previous section, is the solution of Problem 1.

It should be noted that the interpolation spline (1.4), the solution of Problem 1, is exact for any polynomials of degree $m-3$ and for trigonometric functions $\sin \omega x$ and $\cos \omega x$.

Now we shall obtain exact formulas for the coefficients of the interpolation spline (1.4). The result is the following

Theorem 4.1. Coefficients of the interpolation spline (1.4), with equally spaced nodes in the space $K_{2}\left(P_{m}\right)$, have the following form

$$
\begin{aligned}
& C_{0}=p\left[C \varphi(0)+\varphi(h)-d_{1}^{-} \sin (h \omega)+d_{2}^{-} \cos (h \omega)+\sum_{\alpha=0}^{m-3} r_{\alpha}^{-} \cdot(-h)^{\alpha}\right]+ \\
& +\sum_{k=1}^{m-1} \frac{A_{k} p}{\lambda_{k}}\left[\sum_{\gamma=0}^{N} \lambda_{k}^{\gamma} \varphi(h \gamma)+M_{k}+\lambda_{k}^{N} N_{k}\right] \\
& C_{\beta}=p[\varphi(h \beta-h)+C \varphi(h \beta)+\varphi(h \beta+h)]+ \\
& +\sum_{k=1}^{m-1} \frac{A_{k} p}{\lambda_{k}}\left[\sum_{\gamma=0}^{N} \lambda_{k}^{|\beta-\gamma|} \varphi(h \gamma)+\lambda_{k}^{\beta} M_{k}+\lambda_{k}^{N-\beta} N_{k}\right], \beta=1,2, \ldots, N-1, \\
& C_{N}=p\left[C \varphi(1)+\varphi(1-h)+d_{1}^{+} \sin (\omega+h \omega)+d_{2}^{+} \cos (\omega+h \omega)+\sum_{\alpha=0}^{m-3} r_{\alpha}^{+} \cdot(1+h)^{\alpha}\right] \\
& +\sum_{k=1}^{m-1} \frac{A_{k} p}{\lambda_{k}}\left[\sum_{\gamma=0}^{N} \lambda_{k}^{N-\gamma} \varphi(h \gamma)+\lambda_{k}^{N} M_{k}+N_{k}\right] \\
& d_{k}=\frac{1}{2}\left(d_{k}^{+}+d_{k}^{-}\right), \quad k=1,2, \quad r_{\alpha}=\frac{1}{2}\left(r_{\alpha}^{+}+r_{\alpha}^{-}\right), \quad \alpha=0,1, \ldots, m-3,
\end{aligned}
$$

where

$$
M_{k}=\frac{\lambda_{k}\left[d_{2}^{-}\left(\cos (h \omega)-\lambda_{1}\right)-d_{1}^{-} \sin (h \omega)\right]}{\lambda_{k}^{2}+1-2 \lambda_{k} \cos (h \omega)}+\sum_{\alpha=1}^{m-3} r_{\alpha}^{-}(-h)^{\alpha} \sum_{i=1}^{\alpha} \frac{\lambda_{k}^{i} \Delta^{i} 0^{\alpha}}{\left(1-\lambda_{k}\right)^{i+1}}+\frac{r_{0}^{-} \lambda_{k}}{1-\lambda_{k}},
$$




$$
\begin{aligned}
N_{k}= & \frac{\lambda_{k}\left[d_{2}^{+}\left(\cos (\omega+h \omega)-\lambda_{k} \cos \omega\right)+d_{1}^{+}\left(\sin (\omega+h \omega)-\lambda_{k} \sin \omega\right)\right]}{\lambda_{k}^{2}+1-2 \lambda_{k} \cos (h \omega)}+ \\
& +\sum_{\alpha=1}^{m-3} r_{\alpha}^{+}\left(\sum_{j=1}^{\alpha} C_{\alpha}^{j} h^{j} \sum_{i=1}^{j} \frac{\lambda_{k}^{i} \Delta^{i} 0^{j}}{\left(1-\lambda_{k}\right)^{i+1}}+\frac{\lambda_{k}}{1-\lambda_{k}}\right)+\frac{r_{0}^{+} \lambda_{k}}{1-\lambda_{k}}
\end{aligned}
$$

and $p, C, A_{k}$ are defined by (2.8),(2.9), $\lambda_{k}$ are the roots of the polynomial (2.11), $\left|\lambda_{k}\right|<1$, $\Delta^{i} 0^{\alpha}=\sum_{l=1}^{i}(-1)^{i-l} C_{i}^{l} l^{\alpha}$, and $d_{k}^{-}, d_{k}^{+}, k=1,2, r_{\alpha}^{-}, r_{\alpha}^{+}, \alpha=0,1, \ldots, m-3$, are defined from the system (4.3), (4.4), (4.6), (4.7).

Proof. First we find the expressions for $d_{2}^{-}$and $d_{2}^{+}$. When $\beta=0$ and $\beta=N$ from (3.15) for $d_{2}^{-}$and $d_{2}^{+}$we get

$$
\begin{aligned}
& d_{2}^{-}=\varphi(0)-r_{0}^{-} \\
& d_{2}^{+}=\frac{\varphi(1)}{\cos \omega}-d_{1}^{+} \tan \omega-\frac{1}{\cos \omega} \sum_{\alpha=0}^{m-3} r_{\alpha}^{+}
\end{aligned}
$$

Now we have $2 m-2$ unknowns $d_{1}^{-}, d_{1}^{+}, r_{\alpha}^{-}, r_{\alpha}^{+}, \alpha=0,1, \ldots, m-3$.

From equation (3.10), by choosing $\beta=-1,-2, \ldots,-(m-1)$ and $\beta=N+1, N+2, \ldots, N+$ $m-1$, we are able to solve the previous system.

Taking into account (3.15), (4.3) and (4.4), from (3.14) we get the following system

$$
\begin{aligned}
& -d_{1}^{-}\left[\sum_{\gamma=1}^{\infty} D_{m}(h \beta+h \gamma) \sin (h \omega \gamma)\right]+\sum_{\alpha=1}^{m-3} r_{\alpha}^{-}\left[(-h)^{\alpha} \sum_{\gamma=1}^{\infty} D_{m}(h \beta+h \gamma) \gamma^{\alpha}\right]+ \\
& +r_{0}^{-}\left[\sum_{\gamma=1}^{\infty} D_{m}(h \beta+h \gamma)(1-\cos (h \omega \gamma))\right]+d_{1}^{+}\left[\sum_{\gamma=1}^{\infty} D_{m}(h(N+\gamma)-h \beta) \frac{\sin (h \omega \gamma)}{\cos \omega}\right]+ \\
& +\sum_{\alpha=1}^{m-3} r_{\alpha}^{+}\left[\sum_{j=1}^{\alpha} C_{\alpha}^{j} h^{j} \sum_{\gamma=1}^{\infty} D_{m}(h(N+\gamma)-h \beta) \gamma^{j}+\sum_{\gamma=1}^{\infty} D_{m}(h(N+\gamma)-h \beta) \frac{\cos \omega-\cos (\omega+h \omega \gamma)}{\cos \omega}\right]+ \\
& +r_{0}^{+}\left[\sum_{\gamma=1}^{\infty} D_{m}(h(N+\gamma)-h \beta) \frac{\cos \omega-\cos (\omega+h \omega \gamma)}{\cos \omega}\right]=-\sum_{\gamma=0}^{N} D_{m}(h \beta-h \gamma) \varphi(h \gamma)- \\
& -\varphi(0)\left[\sum_{\gamma=1}^{\infty} D_{m}(h \beta+h \gamma) \cos (h \omega \gamma)\right]-\frac{\varphi(1)}{\cos \omega}\left[\sum_{\gamma=1}^{\infty} D_{m}(h(N+\gamma)-h \beta) \cos (\omega+h \omega \gamma)\right],
\end{aligned}
$$

where $\beta=-1,-2, \ldots,-(m-1)$ and $\beta=N+1, N+2, \ldots, N+m-1$.

Now we consider the cases $\beta=-1,-2, \ldots,-(m-1)$. From (4.5) replacing $\beta$ by $-\beta$ and using (2.7) and (2.5), after some calculations for $\beta=1,2, \ldots, m-1$, we get the following system of $m-1$ linear equations

where

$$
d_{1}^{-} B_{\beta}^{-}+\sum_{\alpha=0}^{m-3} r_{\alpha}^{-} B_{\beta \alpha}^{-}+d_{1}^{+} B_{\beta}^{+}+\sum_{\alpha=0}^{m-3} r_{\alpha}^{+} B_{\beta \alpha}^{+}=T_{\beta}, \quad \beta=1,2, \ldots, m-1,
$$

$$
B_{\beta}^{-}=-\left[\sum_{k=1}^{m-1} \frac{A_{k}}{\lambda_{k}} \sum_{\gamma=1}^{\infty} \lambda_{k}^{|\beta-\gamma|} \sin (h \omega \gamma)+\sin (h \omega(\beta-1))+C \sin (h \omega \beta)+\sin (h \omega(\beta+1))\right],
$$




$$
\begin{aligned}
B_{\beta \alpha}^{-}= & (-h)^{\alpha}\left[\sum_{k=1}^{m-1} \frac{A_{k}}{\lambda_{k}} \sum_{\gamma=1}^{\infty} \lambda_{k}^{|\beta-\gamma|} \gamma^{\alpha}+(\beta-1)^{\alpha}+C \beta^{\alpha}+(\beta+1)^{\alpha}\right], \\
B_{\beta 0}^{-}= & \sum_{k=1}^{m-1} \frac{A_{k}}{\lambda_{k}} \sum_{\gamma=1}^{\infty} \lambda_{k}^{|\beta-\gamma|}(1-\cos (h \omega \gamma))+(1-\cos (h \omega(\beta-1)))+ \\
& \quad+C(1-\cos (h \omega \beta))+(1-\cos (h \omega(\beta+1))), \\
B_{\beta}^{+}= & \frac{1}{\cos \omega} \sum_{k=1}^{m-1} \frac{A_{k} \lambda_{k}^{N+\beta} \sin (h \omega)}{\lambda_{k}^{2}+1-2 \lambda_{k} \cos (h \omega)}, \\
B_{\beta \alpha}^{+}= & \sum_{k=1}^{m-1} \frac{A_{k} \lambda_{k}^{N+\beta}}{\lambda_{k}}\left[\sum_{j=1}^{\alpha} C_{\alpha}^{j} h^{j} \sum_{i=1}^{j} \frac{\lambda_{k}^{i} \Delta^{i} 0^{j}}{\left(1-\lambda_{k}\right)^{i+1}}+\frac{\lambda_{k}}{1-\lambda_{k}}-\frac{\lambda_{k}\left[\cos (\omega+h \omega)-\lambda_{k} \cos (h \omega)\right]}{\cos \omega\left[\lambda_{k}^{2}+1-2 \lambda_{k} \cos (h \omega)\right]}\right] \\
B_{\beta 0}^{+}= & \sum_{k=1}^{m-1} A_{k} \lambda_{k}^{N+\beta}\left[\frac{1}{1-\lambda_{k}}-\frac{\cos (\omega+h \omega)-\lambda_{k} \cos (h \omega)}{\cos \omega\left[\lambda_{k}^{2}+1-2 \lambda_{k} \cos (h \omega)\right]}\right] \\
T_{\beta}= & -\sum_{k=1}^{m-1} A_{k} \lambda_{k}^{\beta-1} \sum_{\gamma=0}^{N} \lambda_{k}^{\gamma} \varphi(h \gamma)- \\
- & \varphi(0)\left[\sum_{k=1}^{m-1} \frac{A_{k}}{\lambda_{k}} \sum_{\gamma=1}^{\infty} \lambda_{k}^{|\beta-\gamma|} \cos (h \omega \gamma)+\cos (h \omega(\beta-1))+C \cos (h \omega \beta)+\cos (h \omega(\beta+1))\right]- \\
& -\frac{\varphi(1)}{\cos \omega} \sum_{k=1}^{m-1} \frac{A_{k} \lambda_{k}^{N+\beta}\left[\cos (\omega+h \omega)-\lambda_{k} \cos \omega\right]}{\lambda_{k}^{2}+1-2 \lambda_{k} \cos (h \omega)} .
\end{aligned}
$$

Here $\beta=1,2, \ldots, m-1$ and $\alpha=1,2, \ldots, m-3$.

Further, in (4.5), we consider the cases $\beta=N+1, N+2, \ldots, N+m-1$. From (4.5) replacing $\beta$ by $N+\beta$ and using (2.7) and (2.5), after some calculations for $\beta=1,2, \ldots, m-1$ we get the following system of $m-1$ linear equations

$$
d_{1}^{-} A_{\beta}^{-}+\sum_{\alpha=0}^{m-3} r_{\alpha}^{-} A_{\beta \alpha}^{-}+d_{1}^{+} A_{\beta}^{+}+\sum_{\alpha=0}^{m-3} r_{\alpha}^{+} A_{\beta \alpha}^{+}=S_{\beta}, \quad \beta=1,2, \ldots, m-1,
$$

where

$$
\begin{gathered}
A_{\beta}^{-}=-\sum_{k=1}^{m-1} \frac{A_{k} \lambda_{k}^{N+\beta} \sin (h \omega)}{\lambda_{k}^{2}+1-2 \lambda_{k} \cos (h \omega)}, \\
A_{\beta \alpha}^{-}=(-h)^{\alpha} \sum_{k=1}^{m-1} A_{k} \lambda_{k}^{N+\beta-1} \sum_{i=1}^{\alpha} \frac{\lambda_{k}^{i} \Delta^{i} 0^{\alpha}}{\left(1-\lambda_{k}\right)^{i+1}}, \\
A_{\beta 0}^{-}=\sum_{k=1}^{m-1} \frac{A_{k} \lambda_{k}^{N+\beta}\left(\lambda_{k}+1\right)(\cos (h \omega)-1)}{\left(\lambda_{k}-1\right)\left(\lambda_{k}^{2}+1-2 \lambda_{k} \cos (h \omega)\right)} \\
A_{\beta}^{+}=\frac{1}{\cos \omega}\left[\sum_{k=1}^{m-1} \frac{A_{k}}{\lambda_{k}} \sum_{\gamma=1} \lambda_{k}^{|\beta-\gamma|} \sin (h \omega \gamma)+\sin (h \omega(\beta-1))+C \sin (h \omega \beta)+\sin (h \omega(\beta+1))\right], \\
A_{\beta \alpha}^{+}=\sum_{j=1}^{\alpha} C_{\alpha}^{j} h^{j}\left[\sum_{k=1}^{m-1} \frac{A_{k}}{\lambda_{k}} \sum_{\gamma=1}^{\infty} \lambda_{k}^{|\beta-\gamma|} \gamma^{j}+(\beta-1)^{j}+C \beta^{j}+(\beta+1)^{j}\right]+ \\
+\sum_{k=1}^{m-1} \frac{A_{k}}{\lambda_{k}} \sum_{\gamma=1}^{\infty} \lambda_{k}^{|\beta-\gamma|}+2+C-\frac{1}{\cos \omega}\left[\sum_{k=1}^{m-1} \frac{A_{k}}{\lambda_{k}} \sum_{\gamma=1}^{\infty} \lambda_{k}^{|\beta-\gamma|} \cos (\omega+h \omega \gamma)+\right.
\end{gathered}
$$




$$
\begin{gathered}
+\cos (\omega+h \omega(\beta-1))+C \cos (\omega+h \omega \beta)+\cos (\omega+h \omega(\beta+1))], \\
A_{\beta 0}^{+}=\sum_{k=1}^{m-1} \frac{A_{k}}{\lambda_{k}} \sum_{\gamma=1}^{\infty} \lambda_{k}^{|\beta-\gamma|}+2+C-\frac{1}{\cos \omega}\left[\sum_{k=1}^{m-1} \frac{A_{k}}{\lambda_{k}} \sum_{\gamma=1}^{\infty} \lambda_{k}^{|\beta-\gamma|} \cos (\omega+h \omega \gamma)+\right. \\
+\cos (\omega+h \omega(\beta-1))+C \cos (\omega+h \omega \beta)+\cos (\omega+h \omega(\beta+1))], \\
S_{\beta}=-\sum_{k=1}^{m-1} \frac{A_{k}}{\lambda_{k}} \sum_{\gamma=0}^{N} \lambda_{k}^{N+\beta-\gamma} \varphi(h \gamma)-\varphi(0) \sum_{k=1}^{m-1} \frac{A_{k} \lambda_{k}^{N+\beta}\left(\cos (h \omega)-\lambda_{k}\right)}{\lambda_{k}^{2}+1-2 \lambda_{k} \cos (h \omega)}- \\
-\frac{\varphi(1)}{\cos \omega}\left[\sum_{k=1}^{m-1} \frac{A_{k}}{\lambda_{k}} \sum_{\gamma=1}^{\infty} \lambda_{k}^{|\beta-\gamma|} \cos (\omega+h \omega \gamma)+\right. \\
+\cos (\omega+h \omega(\beta-1))+C \cos (\omega+h \omega \beta)+\cos (\omega+h \omega(\beta+1))] .
\end{gathered}
$$

Here $\beta=1,2, \ldots, m-1$ and $\alpha=1,2, \ldots, m-3$.

Thus for the unknowns $d_{1}^{-}, d_{1}^{+}, r_{\alpha}^{-}, r_{\alpha}^{+}, \alpha=0,1, \ldots, m-3$ we have obtained system (4.6), (4.7) of $2 m-2$ linear equations. Since our interpolation problem has a unique solution, the main matrix of this system is non singular. Unknowns $d_{1}^{-}, d_{1}^{+}, r_{\alpha}^{-}, r_{\alpha}^{+}, \alpha=0,1, \ldots, m-3$ can be found from system (4.6), (4.7). Then taking into account (3.16), using (4.3) and (4.4) we have $d_{k}=\frac{1}{2}\left(d_{k}^{+}+d_{k}^{-}\right), k=1,2, r_{\alpha}=\frac{1}{2}\left(r_{\alpha}^{+}+r_{\alpha}^{-}\right), \alpha=0,1, \ldots, m-3$. Now we find the coefficients $C_{\beta}, \beta=0,1, \ldots, N$.

From (3.6), taking into account (3.15), we deduce

$$
\begin{aligned}
C_{\beta}= & \sum_{\gamma=0}^{N} D_{m}(h \beta-h \gamma) \varphi(h \gamma)+ \\
& +\sum_{\gamma=1}^{\infty} D_{m}(h \beta+h \gamma)\left[d_{1}^{-} \sin (-h \omega \gamma)+d_{2}^{-} \cos (h \omega \gamma)+\sum_{\alpha=0}^{m-3} r_{\alpha}^{-}(-h \gamma)^{\alpha}\right]+ \\
& +\sum_{\gamma=1}^{\infty} D_{m}(h(N+\gamma)-h \beta)\left[d_{1}^{+} \sin (\omega+h \omega \gamma)+d_{2}^{+} \cos (\omega+h \omega \gamma)+\sum_{\alpha=0}^{m-3} r_{\alpha}^{+}(1+h \gamma)^{\alpha}\right],
\end{aligned}
$$

where $\beta=0,1, \ldots, N$.

From here, using (2.7) and formula (2.5), taking into account (4.1) and (4.2), after some calculations we arrive at the expressions of the coefficients $C_{\beta}, \beta=0,1, \ldots, N$ which are given in the assertion of the theorem. Theorem 4.1 is proved.

Remark. From Theorem 4.1, when $m=2$, we get Theorem 7 of [17] and Theorem 3.1 of [19], and when $m=2, \omega=1$ we get Theorem 3.1 of [18].

\section{References}

[1] J.H.Ahlberg, E.N.Nilson, J.L.Walsh, The theory of splines and their applications, Mathematics in Science and Engineering, New York, Academic Press, 1967.

[2] R.Arcangeli, M.C.Lopez de Silanes, J.J.Torrens, Multidimensional minimizing splines, Kluwer Academic publishers, Boston, 2004. 
[3] M.Attea, Hilbertian kernels and spline functions, Studies in Computational Matematics 4, C. Brezinski and L. Wuytack eds, North-Holland, 1992.

[4] A.Berlinet, C.Thomas-Agnan, Reproducing Kernel Hilbert Spaces in Probability and Statistics, Kluwer, Dordrecht, 2004.

[5] B.D.Bojanov, H.A.Hakopian, A.A.Sahakian, Spline functions and multivariate interpolations, Kluwer, Dordrecht, 1993.

[6] C. de Boor, Best approximation properties of spline functions of odd degree, J. Math. Mech., 12(1963), 747-749.

[7] C. de Boor, A practical guide to splines, Springer-Verlag, 1978.

[8] A.Cabada, A.R.Hayotov, Kh.M.Shadimetov, Construction of $D^{m}$-splines in $L_{2}^{(m)}(0,1)$ space by Sobolev method, Applied Mathematics and Computation, 244(2014), 542-551.

[9] J.Duchon, Splines minimizing rotation-invariant semi-norms in Sobolev spaces, in: Constructive Theory of Functions of Several Variables, Lecture Notes in Mathematics, 571 (1977), 85-100.

[10] R.L.Eubank, Spline smoothing and nonparametric regression, Marcel-Dekker, New-York, 1988.

[11] W.Freeden, Spherical spline interpolation-basic theory and computational aspects, Journal of Computational and Applied Mathematics, 1(1984), 367-375.

[12] W.Freeden, Interpolation by multidimensional periodic splines, Journal of Approximation Theory, 55(1988), 104-117 .

[13] P.J.Green, B.W.Silverman, Nonparametric regression and generalized linear models. A roughness penalty approach, Chapman and Hall, London, 1994.

[14] M.Golomb, Approximation by periodic spline interpolants on uniform meshes, Journal of Approximation Theory, 1(1968), 26-65.

[15] R.W.Hamming, Numerical Methods for Scientists and Engineers, Second edition, New York, McGraw-Hill, 1973.

[16] A.R.Hayotov, The discrete analogue of the differential operator $\frac{d^{2 m}}{d x^{2 m}}+2 \omega^{2} \frac{d^{2 m-2}}{d x^{2 m-2}}+$ $2 \omega^{4} \frac{d^{2 m-4}}{d x^{2 m-4}}$, arXiv:1310.6831v1 [math.NA] 25 October (2013).

[17] A.R.Hayotov, The discrete analogue of a differential operator and its applications, Lithuanian Mathematical Journal, 54(2014), no. 3, 290-307.

[18] A.R.Hayotov, G.V.Milovanović, Kh.M.Shadimetov, Interpolation splines minimizing a seminorm, Calcolo, 51(2014), no. 2, 245-260.

[19] A.R.Hayotov, G.V.Milovanović, Kh.M.Shadimetov. Optimal quadrature fromulas and interpolation splines minimizing the semi-norm in the Hilbert space $K_{2}\left(P_{2}\right)$. G.V. Milovanović and M.Th.Rassias (eds.), Analytic Number Theory, Approximation Theory, and Special Functions, 2014, 572-611, doi: 10.1007/978-1-4939-0258-3__22. 
[20] J.C.Holladay, Smoothest curve approximation, Math. Tables Aids Comput., 11(1957), 223-243.

[21] M.I.Ignatev, A.B.Pevniy, Natural splines of many variables, Nauka, Leningrad, 1991 (in Russian).

[22] P.Köhler, On the weights of Sard's quadrature formulas. Calcolo, 25(1988), 169-186 .

[23] N.P.Korneichuk, V.F.Babenko, A.A.Ligun, Extremal properties of polynomials and splines, Naukova dumka, Kiev, 1992 (in Russian).

[24] P.-J.Laurent, Approximation and Optimization, Mir, Moscow, 1975 (in Russian).

[25] N.H.Mamatova, A.R.Hayotov, Kh.M.Shadimetov, Construction of lattice optimal interpolation formulas in Sobolev space of $n$ variable periodic functions by Sobolev method, Ufa Matematical Journal, 5(2013), no. 1, 90-101.

[26] G.Mastroianni, G.V.Milovanović, Interpolation Processes - Basic Theory and Applications, Springer Monographs in Mathematics, Springer - Verlag, Berlin - Heidelberg, 2008.

[27] G.Nürnberger, Approximation by Spline Functions, Springer, Berlin, 1989.

[28] I.J.Schoenberg, On trigonometric spline interpolation, J. Math. Mech., 13(1964), 795-825.

[29] L.L.Schumaker, Spline functions: basic theory, J. Wiley, New-York, 1981.

[30] Kh.M.Shadimetov, Optimal formulas of approximate integration for differentiable functions, Candidate dissertation, Novosibirsk, 1983 (arXiv:1005.0163v1 [math.NA], 2010) (in Russian).

[31] Kh.M.Shadimetov, A.R.Hayotov, Construction of interpolation splines minimizing seminorm in $W_{2}^{(m, m-1)}(0,1)$ space, BIT Numer Math., 53(2013), no. 2, 545-563.

[32] Kh.M.Shadimetov, A.R.Hayotov, S.S.Azamov, Interpolation splines minimizing semi-norm in $K_{2}\left(P_{2}\right)$ space, American Journal of Numerical Analysis, 2(2014), no. 4, 107-114.

[33] S.L.Sobolev, The coefficients of optimal quadrature formulas, in: Selected Works of S. L. Sobolev, Springer, 2006, 561-566.

[34] S.L.Sobolev, Introduction to the Theory of Cubature Formulas, Nauka, Moscow, 1974 (in Russian).

[35] S.L.Sobolev, V.L.Vaskevich, The Theory of Cubature Formulas, Kluwer Academic Publishers Group, Dordrecht 1997.

[36] S.B.Stechkin, Yu.N.Subbotin, Splines in computational mathematics, Nauka, Moscow, 1976 (in Russian).

[37] V.A.Vasilenko, Spline functions: Theory, Algorithms, Programs, Nauka, Novosibirsk, 1983 (in Russian).

[38] G.Wahba, Spline models for observational data, CBMS 59, SIAM, Philadelphia, 1990. 


\section{Построение интерполяционных сплайнов, минимизирую- щих полунорму в пространстве $K_{2}\left(P_{m}\right)$}

Абдулло Р. Хаётов

Институт математики им. В.И. Романовского

Академия наук Республики Узбекистан М. Улугбека 81, Ташкент, 100125

Узбекистан

В настоящей статъе, используя метод С.Л. Соболева, построены интерполячионные сплайны, минимизирующие выражения $\int_{0}^{1}\left(\varphi^{(m)}(x)+\omega^{2} \varphi^{(m-2)}(x)\right)^{2} d x$ в пространстве $K_{2}\left(P_{m}\right)$. Получены явные формулы для коэфбициентов интерполяционных сплайнов. Построенные интерполячионные формуль точны для одночленов $1, x, x^{2}, \ldots, x^{m-3}$ и тригонометрических функиий $\sin \omega x$ u $\cos \omega x$.

Ключевые слова: интерполяционный сплайн, гильбертово пространство, свойство минимизации нормы, метод Соболева, функиии дискретного аргумента. 